\title{
Theoretical Aspects of Galaxy Dynamics Using Planetary Nebulae
}

\author{
Anne Mathieu \\ Instituto de Astrofisica de Andalucia, c/ Camino Bajo de Huétor 24, \\ Granada 18080, Spain
}

\section{Introduction}

The dynamics of the outer regions of elliptical galaxies is still poorly known. Their lack of gas means that it is not possible to use the usual optical and $21 \mathrm{~cm}$ emission lines to determine the kinematics out to large radii, and the faintness of their stellar body means that data calculated using absorption lines are limited to the inner part of the galaxy. It is only by measuring the kinematics out to several optical scalelengths that one gets any unambiguous measure of the dark halo properties (Kent 1986).

Fortunately, there is a kinematic tracer that can be used to measure the kinematics in elliptical galaxies out to large radii. Planetary nebulae $(\mathrm{PNe})$ exist in abundance in these galaxies. They can be readily identified by their strong emission lines, and these lines also provide an easy measure of their kinematics. Their spatial distribution follows the underlying light distribution of the galaxy and their luminosities are not correlated with their position. Thus one can still detect a sufficient number of $\mathrm{PNe}$ at large radii and measure their radial velocities to get a radially-extended kinematic mapping of the system.

Using these bright discrete objects as kinematical tracers appears as one of the most promising avenues for exploring the dynamics of the outer parts of elliptical galaxies. As new large planetary nebulae data sets will soon be available with the dedicated instruments such as the Planetary Nebulae Spectrograph, it becomes important to develop new modeling tools to rigorously analyze and interpret the data.

To draw robust dynamical conclusions about the properties of a system, the models should not assume any particular orbital structure and should fully reflect the geometry of the system under study. Until now, elliptical galaxies have usually been described using simple spherical models though these objects have long be indisputably recognized as triaxial. The triaxial nature of a stellar system produces unambiguous signatures in the kinematics that can not be reproduced with a spherical model. Nowadays with increasingly detailed observations of galaxies showing triaxial characteristics, it is time to develop tools for rigorously exploiting the information contained in the data.

To this end, we have developed a dynamical modeling method for triaxial systems which we have applied to the kinematic data of the planetary nebulae of the giant elliptical galaxy Cen A. With a sample of hundreds of planetary nebulae we have built a detailed dynamical model for this galaxy, enabling us to constrain its intrinsic properties such as the shape and mass distributions and study its orbital structure. 


\section{Observations, modeling method and results}

The observational constraints on Cen A include the stellar surface brightness and the velocities of over 400 planetary nebulae from the survey of Hui et al. (1995). The Quadratic Programming method, pioneered by Dejonghe (1989), has since been largely used to built various kinds of dynamical models of stellar systems with spherical and also axisymmetric geometry. We have extended this robust method to the more general and complex case of triaxial models. As such, our modeling technique is to date the only method available to properly treat the dynamics of elliptical galaxies. To this end we have developed a whole new set of basic distribution functions (the so-called Abel components) using triaxial gravitational potentials of Stäckel form. A great advantage of using this kind of potential is that the moments of the distribution function can be calculated analytically. Furthermore we have shown that these gravitational potentials are realistic approximations of the potentials of real galaxies (Mathieu \& Dejonghe 1996).

Using both the stellar photometry and the PN kinematic data, we show that a model with constant mass-to-light ratio can not fit the data, thereby implying the presence of a dark halo in this galaxy. The best-fit triaxial model yields a good fit to the photometry and to the planetary nebulae kinematic data (rotation and velocity dispersion along the major and minor axis of the galaxy). The model successfully reproduces the triaxial signature of the PN rotation and dispersion data. With this model the luminous and dark mass distributions and shape can be well constrained. Quantitative details of the model can be found in Mathieu \& Dejonghe (1999).

\section{Conclusions}

We have described a new method for modeling galaxies using the kinematics of discrete objects such as PNe. Our application of the triaxial modeling to Cen A proves that the method is fully effective at analyzing the dynamical structure of complex stellar systems in great details. As more PN data in external galaxies are becoming available, this modeling method will undoubtedly be an indispensable tool for exploring the dynamics of galaxies.

\section{References}

Dejonghe, H. 1989, ApJ, 343, 113

Hui, X., Ford, H. C., Freeman, K. C., \& Dopita, M. A. 1995, ApJ, 449, 592

Kent, S. M. 1986, AJ, 91, 1301

Mathieu, A., \& Dejonghe, H. 1996, A\&A, 314, 25

Mathieu, A., \& Dejonghe, H. 1999, MNRAS, 303, 455 